\title{
The broad line emission from the AGN 3C 390.3: complex broad-line region and perturbation in accretion disk
}

\author{
Ilić Dragana ${ }^{1}$, Luka Č. Popović ${ }^{2}$, Alla I. Shapovalova ${ }^{3}$, \\ Alexander N. Burenkov ${ }^{3}$, Vahram H. Chavushyan ${ }^{4}$, \\ Andjelka Kovačević ${ }^{1}$ and Wolfram Kollatschny ${ }^{5}$ \\ ${ }^{1}$ Department of Astronomy, Faculty of Mathematics, University of Belgrade, \\ Studentski trg 16, 11000 Belgrade, Serbia, email: dilic@matf.bg.ac.rs \\ ${ }^{2}$ Astronomical Observatory, Volgina 7, 11160 Belgrade 74, Serbia \\ ${ }^{3}$ Special Astrophysical Observatory of the Russian AS, Nizhnij Arkhyz, \\ Karachaevo-Cherkesia 369167, Russia \\ ${ }^{4}$ Instituto Nacional de Astrofísica, Óptica y Electrónica, Apartado Postal 51, \\ CP 72000, Puebla, Pue. México \\ ${ }^{5}$ Institut für Astrophysik, Friedrich-Hund-Platz 1, Göttingen, Germany
}

\begin{abstract}
From 13-years of the spectral optical monitoring of a well-known broad-line radio galaxy 3C 390.3 we concluded that the geometry of the broad emission-line region is complex, while still the main part of the emission is coming from the accretion disk. Here we present part of the analysis of the broad $\mathrm{H} \alpha$ and $\mathrm{H} \beta$ emission lines, which are showing highly variable double-peaked profiles during the monitoring period (1995-2007), with the aim to probe the accretion disk properties. The disk-like geometry plays a dominant role, but the variability of $\mathrm{H} \alpha$ and $\mathrm{H} \beta$ line profiles and intensities shows a presence of an additional emission-line region, that has a different nature for different periods, e.g. in one period the observed variation can be well modeled if one assumes changes in position and size of the emitting disk along the accretion disk.
\end{abstract}

Keywords. Galaxies: active, galaxies: quasar: individual (3C 390.3), line: profiles

\section{Introduction}

The long-term spectral monitoring in the optical band is a powerful tool for studying the structure of active galactic nuclei (AGN), especially of the broad line region (BLR), as it has been shown that the broad emission lines, the main signature of the BLR, are strongly variable. One of the object in our more than a decade long monitoring campaign of type 1 AGN (see Shapovalova et al. 2009 for a review) is a well known broad-line radio galaxy 3C 390.3 (Shapovalova et al. 2010b, Popović et al. 2011). For details about the monitoring campaign, data analysis and results see Shapovalova et al. (2004), Shapovalova et al. (2008), Shapovalova et al. (2010a), Shapovalova et al. (2010b), Popović et al. (2011). In a similar way to about $10 \%$ of radio-loud AGN, 3C 390.3 emits very broad, double-peaked emission lines, whose fluxes and line profile parameters are drastically changing in the monitoring period. We can basically divide the monitoring period into two: period I - until 2002, and period II - after 2002, when there is a clear outburst seen in light curves (see Fig. 6 in Shapovalova et al. 2010b). Here we give some results coming from the analysis of the variability of the broad $\mathrm{H} \alpha$ and $\mathrm{H} \beta$ double-peaked emission lines, especially about the geometry of the BLR (for more details see Popović et al. 2011). 

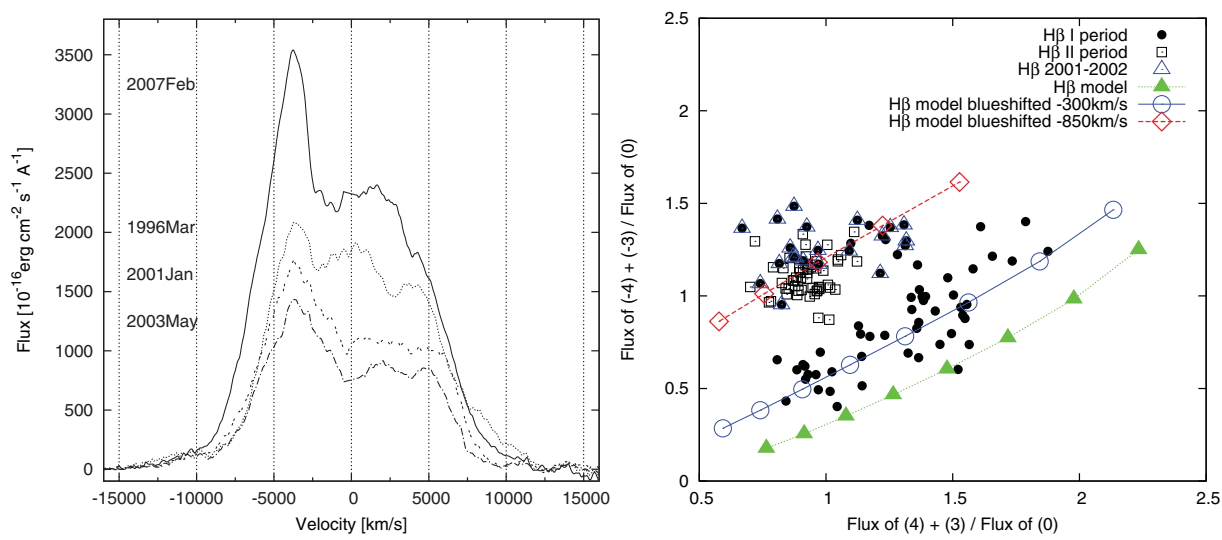

Figure 1. Left: Some examples of the month-averaged profiles of the $\mathrm{H} \alpha$ broad emission line. Right: The blue-wing flux (two blue line segments, -4 and -3 ) vs. the red-wing (two red line segments, +4 and +3 ) of $\mathrm{H} \beta$, both normalized to the central line segment flux. Observations in period I are denoted with full circles, in period II with open squares, while observations from 2001-2002 are marked with open triangles. Notation of each curve is given (Popović et al. 2011).

\section{Some results}

The line profiles are changing during the monitoring period (Fig. 1, left), always showing the disk-like profile with the more pronounced blue peak. But, there is also the central peak (Fig. 1, left) that may come from the emission region additional to the accretion disk, or it may be caused by disk perturbation (Jovanović et al. 2010). We divide the line profiles along the velocity scale into segments, so that line segments -4 and -3 represent together the blue wing flux and line segments 4 and 3 the red wing. We focus on the analysis of the line wings, since for them the emission of the accretion disk is contributing the most. For the line profiles modeling, we used the Keplerian disk model and the input parameters from the work of Flohic \& Eracleous (2008). Then the change in the line profile wings in the period I (full circles, Fig. 1, right), can be explained if the location of the disk emitting the broad lines is shifted along the accretion disk (blue line with open circles, Fig. 1, right), from $R_{\text {inn }}=250 R_{\mathrm{g}}$ to $550 R_{\mathrm{g}}$. However, in the period II (open squares, Fig. 1, right), when the outburst starts, there is no changes in the disk structure, only an outflow in the BLR is present (see Popović et al. 2011 for more details).

\section{Acknowledgements}

This work was supported by INTAS (grant N96-0328), RFBR (grants N97-02-17625 N00-02-16272, N03-02-17123, 06-02-16843, and N09-02-01136), State program Astronomy (Russia), CONACYT research grant 39560-F and 54480 (México) and the Ministry of Education and Science of Republic of Serbia through the project Astrophysical Spectroscopy of Extragalactic Objects \#176001.

\section{References}

Jovanović, P., Popović, L. Č., Stalevski, M., \& Shapovalova, A. I. 2010, ApJ, 718, 168

Flohic, H. M. L. G. \& Eracleous, M. 2008, ApJ, 686, 138

Popović, L. Č., Shapovalova, A. I., Ilić, D. et al. 2011, A\&BA, 528, 130

Shapovalova, A. I., Doroshenko, V. T., Bochkarev, N. G. et al. 2008, A\& A, 422, 925

Shapovalova, A. I., Popović, L. Č., Collin, S. et al. 2008, A\&BA, 486, 99

Shapovalova, A. I., Popović, L. C.., Burenkov, A. N. et al. 2009, NewAR, 53, 191

Shapovalova, A. I., Popović, L. Č., Burenkov, A. N. et al. 2010a, A\&AA, 509, 106

Shapovalova, A. I., Popović, L. C., Burenkov, A. N. et al. 2010b, A\&\&A, 517, 42 